\title{
Free vibration characteristics of thick doubly curved variable stiffness composite laminated shells using higher-order shear deformation theory
}

\author{
Anand Venkatachari ${ }^{* 1,3}, K$. Ramajeyathilagam ${ }^{2}$ \\ ${ }^{1}$ School of Aeronautical Sciences, Hindustan Institute Technology and Science 603103 Chennai, India \\ ${ }^{2}$ School of Aeronautical Sciences, Hindustan Institute Technology and Science 603103 Chennai, India \\ ${ }^{3}$ Tech Mahindra Limited, Electronics city, 560100 Bangalore, India
}

\begin{abstract}
In the present work, the natural frequencies of cylindrical and spherical laminated shells with variable stiffness are numerically studied using a shear flexible isogeometric finite element. The kinematics relies on cubic shear deformation theory in which cubic variation is assumed for the surface displacements and a quadratic variation for the traverse displacement along the thickness. A zig-zag function, used for the in-plane displacements, accounts for the abrupt discontinuity at the boundaries of the laminae. The Lagrangian equations of motion is deployed to solve the frequencies of curved panels. A detailed parametric analysis examines the influence of fibre centre/edge angles, shell geometric variables, material anisotropy and edge conditions on frequencies and mode shapes.
\end{abstract}

\section{Introduction}

Advanced composites have wide application in the fields of aerospace and power generation due to high strength and stiffness relative to its weight. There is always a growing need to increase the pay load and reduce the cost of material and manufacturing in the aerospace industry. The proportion of composites to metals have increased multi fold in two decades in the aerospace industry.

Designers have implemented many ways to improve the performance of composite structures. Few methods of increasing stiffness are curving the fibres on the surface of lamina, increase the volume of fibres relative to matrix, tow drops and addition of stiffeners. A novel idea of curving the fibres continuously on the surface of lamina has emerged few years ago and these fibres are called curvilinear or curved fibres. The variation in stiffness on the surface of the lamina owing to variation in the fibre direction and these laminates form a part of Variable Stiffness Composite Laminates (VSCL) or curved fibre composite laminates. Many researchers have shown heightened interest in the investigation of structural response of the curved fibre composites, thanks to the advances in the manufacturing technology. The fibres remain straight in traditional laminates, though different lamina can have different orientation. Such laminates are termed as Constant Stiffness Composite Laminates (or CSCL).

In general, plate/shell problems deploy linear/nonlinear governing equations and solved by a suitable analytical/ numerical approach. Analytical results assumes deformed shape for prediction of the nonlinear behaviour. Finite element procedure is best suited for the solution of such structures without assuming a mode shape apriori and the solution itself predicts the deformed configuration based on the support conditions. Finite elements using the field-consistency approach coupled with cubic shear deformation kinematic model, developed recently for the structural analyses of thick and thin laminates do not exhibit shear/membrane locking phenomenon and do not require lesser order integration scheme in evaluating the energy terms. The associativity between the CAD and finite elements is a desirable feature in the optimization of the airframe structures. This association between $\mathrm{CAD} / \mathrm{CAE}$ is provided by a novel concept called Iso Geometric Analysis (IGA). The interpolation expressions for the field variables are same as the B-splines of geometry.

The technology of placing fibres automatically is the key technology discovered in 1980's for the fabrication of curved fibre composites. Kim et al. [1] proposed a novel tow placement technique considering the shear capability of tows, thus reducing defects. The normal shear moduli in composites, unlike isotropic material, is less than the surface young's modulus leading to significant shear deformation and hence results in significant transverse shear stress. The CLPT neglects the shear effects in the normal direction and hence not suitable for multi-layered thick or moderately thick plates. It is opined by Kant and Swaminathan [2] that CLPT under-predicts deflections and over-estimates eigenvalues in vibration and buckling problems. The FSDT assumes linear change in deformation through the 
laminate thickness resulting in constant shear stress in normal direction in different laminae. This necessitates adhoc variables that are not easy to compute for all the problems. The limitations of FSDT can be overcome by the use of HSDT that includes more terms from the Taylor series in the kinematics of displacement formulation.

Abdalla et al.[3] dealt with an optimization problem aimed at the increase of frequency of VSCL panels. The CLPT formulation is employed and the stiffness is changed by the parameters of laminate. It is shown that the frequency of a VSCL design is $12 \%$ more than the CSCL. Honda and Narita [4] developed FEM to analyse vibration of plates made from anisotropic properties and optimized the design for vibratory environment. It is shown that continuously varying fibre orientation affects fundamental frequency. The frequency, thus obtained is higher than straight fibres.

Blom et al. [5] optimized the design of VSCL conical panels for maximum eigenvalue. It is shown that the frequency of a particular conical shell can be amplified by $20 \%$ with curved fibres. It is also emphasized that manufacturability has a large influence on the eigenvalue and the manufacturing details should be considered during the design phase. Lo and Hyer [6] studied the eigenvalues of thin-walled structures by changing fibre direction. The varying fibre orientation changes axial buckling load, the effect on the frequency of elliptic/circular cylinders is not significant. Ribeiro [7] investigated linear eigenvalues of thin cylindrical shells using a polynomial type of finite element. A Parametric study examines the role of fibre angles on the modes of vibration. The curved fibre laminates have a larger effect on the eigenvalues in cylindrical shells than in plates. It was noticed that angle at the edge of the fibre has a influence on vibratory behaviour.

The studies, till date, on the vibration/buckling characteristics of curved fibre composite shells with isogeometric FE based on higher-order theories are minimal. In this paper, the vibratory behaviour of curved fibre shells numerically simulated by sophisticated NURBS finite element with cubic deformation theory with zig-zag function.

\section{Theoretical Formulation}

The direction of the cured fibres changes continuously in the surface of the lamina. The stiffness changes due to the variation in the direction of fibres. The shell and the reference fibre passing through the centre is shown in Figures. 1 and 2 respectively. The rest of fibres are created by shifting the fibres relative to the fibre at the centre. The equation for the reference fibre and orientation at any point in the plane along $\mathrm{X}$ direction is given by Houmat [8] as:

$y=\left\{\begin{array}{cc}\frac{a}{2(T 1-T 0)}\left\{-\log _{n}(\cos T 0)+\log _{n}\left[\cos \left(T 0-\frac{2(T 1-T 0) x}{a}\right)\right]\right\} & -a / 2 \leq x \leq 0 \\ \frac{a}{2(T 1-T 0)}\left\{\log _{n}(\cos T 0)-\log _{n}\left[\cos \left(T_{0}+\frac{2(T 1-T 0) x}{a}\right)\right]\right\} & 0 \leq x \leq a / 2\end{array}\right.$

$$
\theta(x)=\left\{\begin{array}{lr}
\frac{-2}{a}(T 1-T 0) x+T 0, & -\frac{a}{2} \leq x \leq 0 \\
\frac{2}{a}(T 1-T 0) x+T 0, & 0 \leq x \leq a / 2
\end{array}\right.
$$

where $(x, y)$ are the coordinates of any point in the surface of the lamina, $T 0, T 1$ are fibre centre/edge angles and $a$ is the plate length and $\theta$ is the angle of the fibre. The curvature of the fibre should be less than $3.28 \mathrm{~m}^{-1}$ for manufacturability as suggested by Houmat [8]. The manufacturable zone of kink-free curved fibre laminates is presented in Figure. 3.

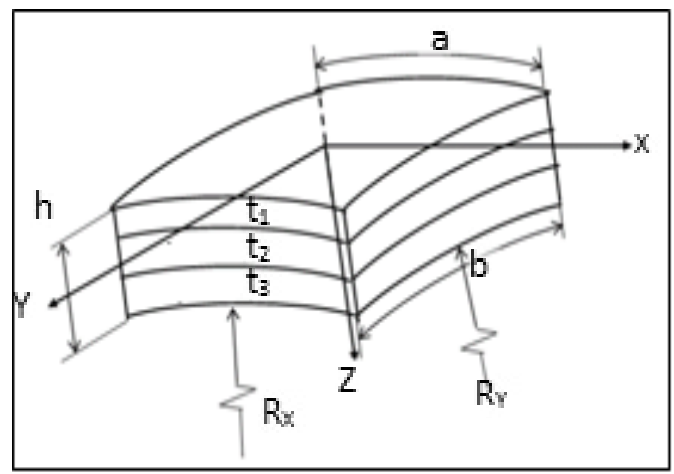

Fig. 1. Shell geometry

The plate/shell kinematics for the $l^{\text {th }}$. layer using cubic kinematic model given by Ganapathi and Makhecha [9] :

$$
\begin{aligned}
& u^{\mathbb{D}}\left(x_{v}, y_{s}, t\right)=\sum_{\mathrm{p}=1}^{5} H_{\mathrm{p}}(z) u_{\mathrm{p}}^{\mathbb{1}}\left(x_{s}, y, t\right) ; \\
& v^{\mathbb{D}}(x, y, z, t)=\sum_{q=1}^{5} H_{q}(z) v_{q}^{\mathbb{D}}\left(x_{v}, y, t\right) \text { : } \\
& w^{\mathbb{1}}(x, y, z, t)=\sum_{\gamma=1}^{\mathrm{a}} H_{\gamma}(z) w_{\gamma}^{\mathbb{1}}(x, y, t)
\end{aligned}
$$

where,

$$
\begin{gathered}
H_{p}(z)= \begin{cases}z^{p-1}, p \leq 4 \\
s^{1}{ }_{s} & p=5^{x}\end{cases} \\
H_{q}(z)= \begin{cases}z^{q-1}, & q \leq 4 \\
S^{1}{ }_{s} & q=5^{x}\end{cases} \\
H_{y}(z)=z^{\gamma-1} \quad r \leq 3_{s}^{x} \\
S^{\mathbb{1}}=2(-1)^{\mathbb{1}} \frac{z_{l}}{h_{l}}
\end{gathered}
$$

Here $u_{1}^{l}, v_{1}^{l}$ and $w_{1}^{l}$ are the displacements on the mid surface. $u_{2}^{l}, v_{2}^{l}$ are the out of surface rotations of normal. $w_{2}^{l}, w_{3}^{l}, u_{3}^{l}, v_{3}^{l}, u_{4}^{l}, v_{4}^{l}$ are the additional variables at the reference plane, arising from higher terms in the Taylor series expansion. $u_{5}^{l}, v_{5}^{l}$ are independent variables multiplied to the zigzag function. 


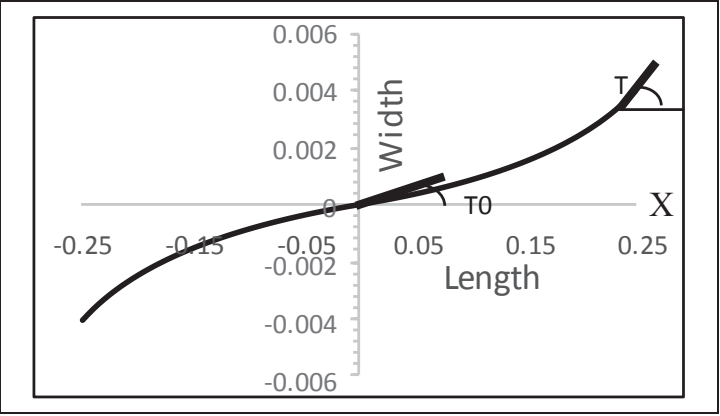

Fig. 2. Reference fibre path

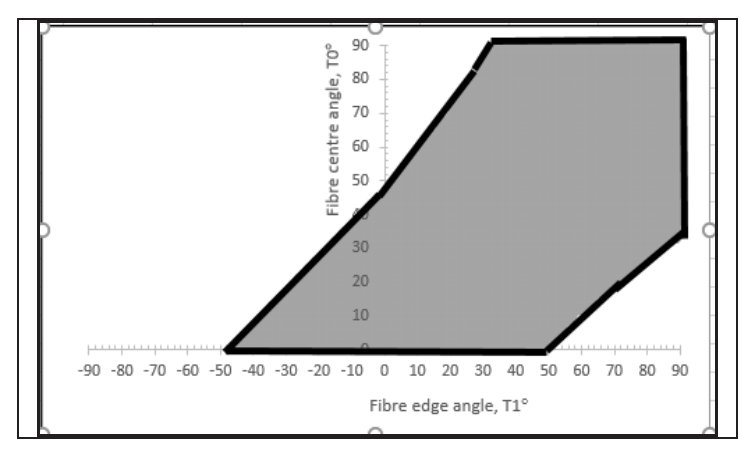

Fig. 3. Fibre angles for manufacturability

The strain is

$$
\varepsilon=\varepsilon^{L}-\varepsilon^{c t}
$$

The strain due to hygro-thermal environment

$$
\varepsilon^{c t}=\Delta T \alpha_{T}+\Delta C \alpha_{C}
$$

where $\Delta \mathrm{T}, \Delta \mathrm{C}$ are change in temperature and moisture and $\alpha_{\mathrm{T}}, \alpha_{\mathrm{C}}$ are their coefficients in the principal directions of the material.

The linear strain is given as:

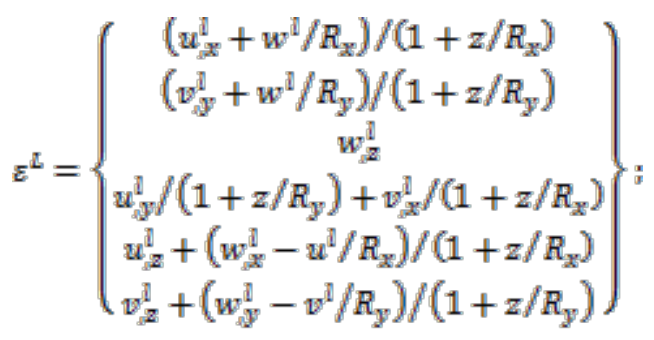

The constitutive equation relating the stress and strain in $l^{\text {th. }}$ lamina,

$$
\sigma=Q^{l} \varepsilon
$$

where $Q^{l}$ is the matrix of stiffness coefficients. The Lagrangian equation of motion, for unknown variables, $\delta_{\mathrm{i}}=\left(\mathrm{u}_{\mathrm{p}}, \mathrm{v}_{\mathrm{q}}, \mathrm{w}_{\mathrm{r}}\right), \mathrm{p}, \mathrm{q}=1$ to 5 and $\mathrm{r}=1$ to 3 is

$$
\frac{d}{d t}\left[\frac{\partial(T-U)}{\partial \delta_{\mathrm{d}}}\right]-\left[\frac{\partial(T-U)}{\partial \delta_{\mathrm{i}}}\right]=0
$$

where $\mathrm{T}$ and $\mathrm{U}$ are kinetic energy and $\mathrm{PE}, i$ being total degrees of freedom. A dot over $\delta_{i}$ indicates the partial derivative of degree of freedom with respect to time. The $\mathrm{KE}$ is given by,

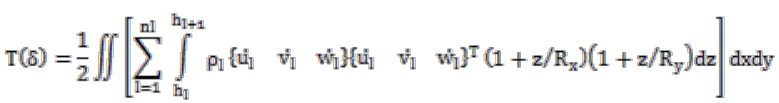

where, $\rho_{1}$ is the mass density of the $l^{\text {th. }}$ layer, $\mathrm{h}_{1}$ and $\mathrm{h}_{1+1}$ are the $\mathrm{z}$ coordinates of the bottom and top layers and $n l$ is the count of layers.

The PE of the shell is

$U(\delta)=\frac{1}{2} \iint\left[\sum_{i=1}^{n l} \sigma^{T} \varepsilon\left(1+z / R_{x}\right)\left(1+z / R_{y}\right) d z\right] d x d y(11)$

The vibratory behaviour using FEM is:

$$
\left[[K]-\omega^{2}[M]\right]\{\delta\}=0
$$

The NURBS FE employs the recurrence formula developed by Mansfield [10]

Let $\mathrm{U}=\left[\mathrm{u}_{0},,,, \mathrm{u}_{\mathrm{m}}\right]$ be a real numbers as per $\mathrm{u}_{\mathrm{i}}<\mathrm{u}_{\mathrm{m}}$ i.e., $\mathrm{u}_{\mathrm{i}} \leq \mathrm{u}_{\mathrm{i}+1}, \mathrm{i}=0, \ldots, \mathrm{m}-1$. These are called knots and $\mathrm{U}$ is $\mathrm{a}$ vector with knots. The basis functions are defined as:

$$
\begin{gathered}
N_{i, p}(u)=\frac{u-u_{i}}{u_{i+p}-u_{i}} N_{i, p-1}(u)+\frac{u_{i+p+1}-u}{u_{i+p+1}-u_{i+1}} N_{i+1, p-1}(u) \\
N_{i, 0}(u)= \begin{cases}1, & \text { if } u_{i} \leq u<u_{i+1} \\
0, & \text { otherwise }\end{cases}
\end{gathered}
$$

A B-spline curve is defined by:

$$
C(u)=\sum_{i=0}^{n} N_{i, p}(u) P_{i} \quad a \leq u \leq b
$$

Where $\left\{\mathrm{P}_{\mathrm{i}}\right\}$ are the control points, and the $\left\{\mathrm{N}_{(\mathrm{i}, \mathrm{p})}(\mathrm{u})\right\}$ are basis functions. The vector with knots is,

$$
U=\left\{a, \ldots, a, u_{p+1}, \ldots, u_{m-p+1}, b, \ldots, b\right\}
$$

$A p^{\text {th }}$ degree NURBS curve is defined by

$$
C(u)=\frac{\sum_{i=0}^{n} N_{i, p}(u) w_{i} P_{i}}{\sum_{i=0}^{n} N_{i, p}(u) w_{i}} \quad a \leq u \leq b
$$

By using the notation

$$
R_{i, p}(u)=\frac{N_{i, p} w_{i}}{\sum_{j=0}^{n} N_{j p p}(u) w_{j}}
$$

Equation (16) can be written as

$$
C(u)=\sum_{i=0}^{n} R_{i, p}(u) P_{i}
$$

The $\left\{\mathrm{R}_{(\mathrm{i}, \mathrm{p})}(\mathrm{u})\right\}$ are the rational basis functions. These are piecewise rational functions on $u \in[0,1]$ as in Fig. 4 .

The NURBS FE with 13 dof per node is used to deduce different lower order models by suppressing dof. The various structural models assessed are listed in Table 1. 
Table 1: First and higher order structural models

\begin{tabular}{|l|l|}
\hline Model & DOF per node \\
\hline HSDT13 & $\begin{array}{l}\mathrm{u} 1, \mathrm{v} 1, \mathrm{w} 1, \mathrm{u} 2, \mathrm{v} 2, \mathrm{w} 2, \mathrm{u} 3, \mathrm{v} 3, \mathrm{w} 3, \mathrm{u} 4, \\
\mathrm{v} 4, \mathrm{u} 5, \mathrm{v} 5\end{array}$ \\
\hline HSDT11A & $\begin{array}{l}\mathrm{u} 1, \mathrm{v} 1, \mathrm{w} 1, \mathrm{u} 2, \mathrm{v} 2, \mathrm{u} 3, \mathrm{v} 3, \mathrm{u} 4, \mathrm{v} 4, \mathrm{u} 5, \\
\mathrm{v} 5\end{array}$ \\
\hline HSDT11B & $\begin{array}{l}\mathrm{u} 1, \mathrm{v} 1, \mathrm{w} 1, \mathrm{u} 2, \mathrm{v} 2, \mathrm{w} 2, \mathrm{u} 3, \mathrm{v} 3, \mathrm{w} 3, \mathrm{u} 4, \\
\mathrm{v} 4\end{array}$ \\
\hline HSDT9 & $\mathrm{u} 1, \mathrm{v} 1, \mathrm{w} 1, \mathrm{u} 2, \mathrm{v} 2, \mathrm{u} 3, \mathrm{v} 3, \mathrm{u} 4, \mathrm{v} 4$ \\
\hline FSDT5 & $u_{1}, v_{1}, w_{1}, u_{2}, v_{2}$ \\
\hline
\end{tabular}

The simple support and clamped edge conditions are as follows:

SS:

$\mathrm{u} 1=\mathrm{w} 1=\mathrm{v} 2=\mathrm{w} 2=\mathrm{w} 3=\mathrm{v} 3=\mathrm{v} 4=\mathrm{v} 5=0$ at $\mathrm{x}= \pm \mathrm{a} / 2$

$\mathrm{v} 1=\mathrm{w} 1=\mathrm{u} 2=\mathrm{w} 2=\mathrm{w} 3=\mathrm{u} 3=\mathrm{u} 4=\mathrm{u} 5=0$ at $\mathrm{y}= \pm \mathrm{b} / 2$

Clamped:

$$
\begin{aligned}
& \mathrm{u}_{1}=\mathrm{v}_{1}=\mathrm{w}_{1}=\mathrm{u}_{2}=\mathrm{v}_{2}=\mathrm{w}_{2}=\mathrm{u}_{3}=\mathrm{v}_{3}=0 \\
& \mathrm{w}_{3}=\mathrm{u}_{4}=\mathrm{v}_{4}=\mathrm{u}_{5}=\mathrm{v}_{5}=\mathrm{u}_{3}=\mathrm{u}_{4}=\mathrm{u}_{5}=0
\end{aligned}
$$

at $\mathrm{x}= \pm a / 2$ and $\mathrm{y}= \pm b / 2 ; a$ and $b$ are length and width respectively.

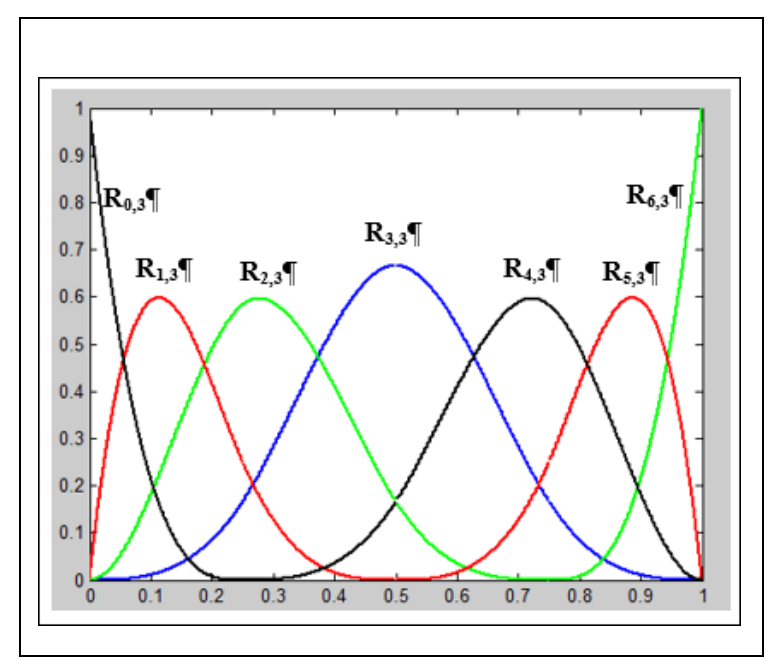

Fig. 4. Rational cubic b-spline functions;

$\mathrm{U}=\{0,0,0,0,1 / 4,1 / 2,3 / 4,1,1,1,1\} ;$ Weights $=\{1,1,1,1,1,1,1\}$

The classical FEM and IGFEM define shape functions differently. The langrange or serendity shape functions is used in classical finite elements while rational B-splines (or NURBS) used to represent geometry in CAD software are used as interpolation functions in isogeometric formulation. The Jacobian defines the transformation from "physical/parent" space in classical finite elements. There is additional space namely parametric space in the IGFEM necessitating the calculation of two jacobians: one for the transformation from physical space to parametric and the second from "parametric/parent" space. The process flow is shown in Figure. 5.

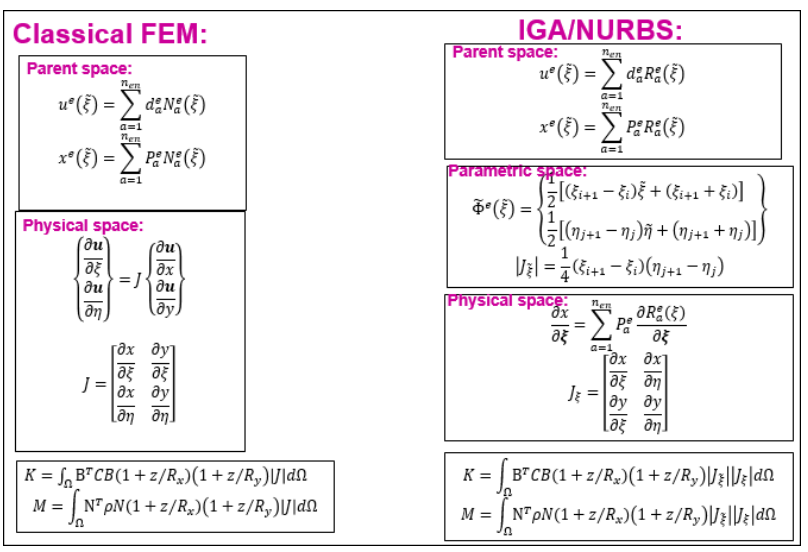

Fig. 5. Flow chart of classical FE and IG FE

\section{Results and Discussion}

The vibratory characteristics of the VSCL shells is assessed with NURBS finite element.

\subsection{Convergence}

The convergence study is performed by systematically decreasing the mesh size. The results from the convergence study are presented in Table 2 .

Table 2: Convergence study of isotropic square plate, $\mathrm{a} / \mathrm{h}=10$,

\begin{tabular}{|l|l|l|l|}
\hline \multicolumn{1}{|c}{$\mathrm{E}=1, v=0.3$} \\
& Mesh Size & Mode 1 & Mode 2 \\
\hline Present & $8 \times 8$ & 0.0930 & 0.2225 \\
Present & $16 \times 16$ & 0.0930 & 0.2219 \\
Present & $32 \times 32$ & 0.0930 & 0.2219 \\
Ref. [11] & & 0.0931 & 0.2221 \\
\hline
\end{tabular}

\subsection{Validation}

The present NURBS FE is validated with the results available in publications.

Table 3 presents the comparison of natural frequencies for spherical and cylindrical shells between the present formulation and Reddy and Liu [12]. It can be noted that the results of the present formulation match very well and the minor difference is due the difference in order of the shear deformation theory. 
Table 3: Non-dimensional frequency for cross-ply, square spherical and cylindrical shells

\begin{tabular}{|c|cc|cc|}
\hline \multirow{3}{*}{$R / a$} & \multicolumn{4}{|c|}{$0^{\circ} / 90^{\circ} / 0^{\circ}$} \\
\cline { 2 - 5 } & $\mathrm{a} / \mathrm{h}=100$ & \multicolumn{2}{c|}{$\mathrm{a} / \mathrm{h}=10$} \\
\cline { 2 - 5 } & Present & Ref. [12] & Present & Ref. [12] \\
\hline \multicolumn{5}{|c|}{ Spherical } \\
\hline 5 & 31.024 & 31.020 & 11.696 & 12.060 \\
10 & 20.387 & 20.350 & 11.521 & 11.860 \\
20 & 16.674 & 16.620 & 11.477 & 11.810 \\
50 & 15.473 & 15.420 & 10.165 & 11.790 \\
100 & 15.294 & 15.240 & 11.462 & 11.790 \\
Plate & 15.234 & 15.170 & 11.462 & 11.790 \\
\hline \multicolumn{5}{|c|}{ Cylindrical } \\
\hline 5 & 20.374 & 20.330 & 11.514 & 11.850 \\
10 & 16.672 & 16.620 & 11.475 & 11.800 \\
20 & 15.606 & 15.550 & 11.465 & 11.791 \\
50 & 15.294 & 15.240 & 11.462 & 11.790 \\
100 & 15.249 & 15.190 & 11.462 & 11.790 \\
Plate & 15.234 & 15.170 & 11.462 & 11.790 \\
\hline
\end{tabular}

\subsection{Parametric study}

A detailed study is performed to assess change in fibre angles, geometric parameters such as different shear deformation theories, side-to-thickness ratio $(\mathrm{a} / \mathrm{h})$, aspect ratio $(\mathrm{a} / \mathrm{b})$, boundary conditions etc. on the eigenvalues and deformed shapes.

\subsubsection{Shear deformation theories}

The different shear deformation models is assessed and shown in Figure. 6. A difference of $8 \%$ is noticed in the frequencies between first and cubic models for thick shells.

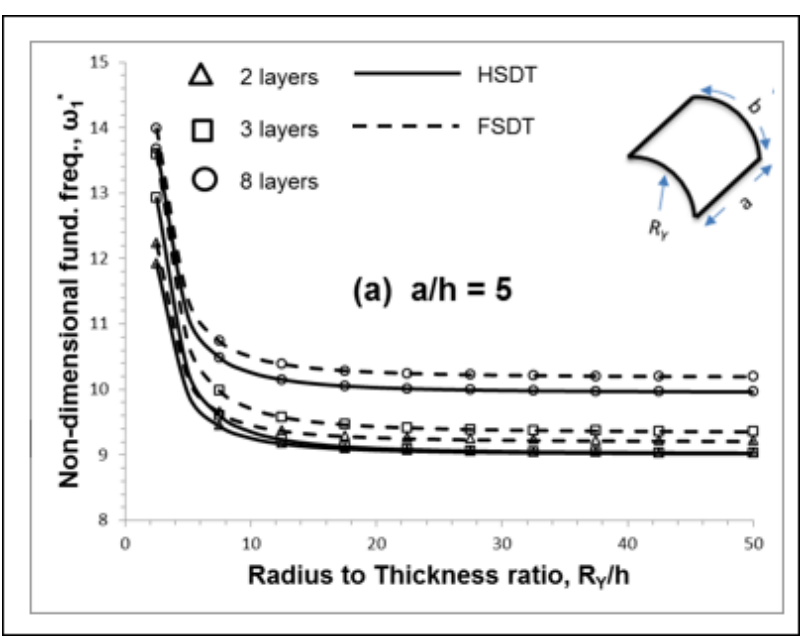

Fig 6: Variation of frequency against the radius-to-thickness of cylindrical 2/3/8-layered shells; T0/T1 $=30^{\circ} / 45^{\circ}$

\subsubsection{Influence of fibre angles}

The effect of fibre centre angle (T0) and the angle at the edge (T1) on the eigenvalues of 4-layered square spherical/cylindrical shells with side-to-thickness ratio, $\mathrm{S}$ of 10 is shown in Figure. 7.
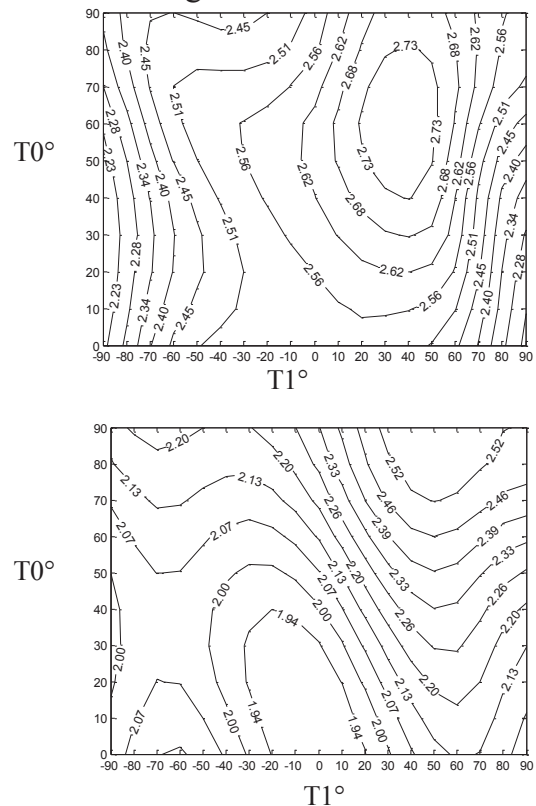

Fig 7: Linear fundamental frequency contours for 4-lay antisymmetric spherical (top) and cylindrical (bot)

The eigenvalue of the spherical shell is observed to be higher than the cylindrical shell due to double curvature. The influence of fibre angles is different for spherical and cylindrical shells. The increase in the fibre centre angle increases the eigenvalues.

\subsubsection{Influence of side-to-thickness ratio}

The frequencies for 2-layered square simply supported shells of different side-to-thickness ratio's and varying fibre centre/edge angles is presented in Figure. 8.

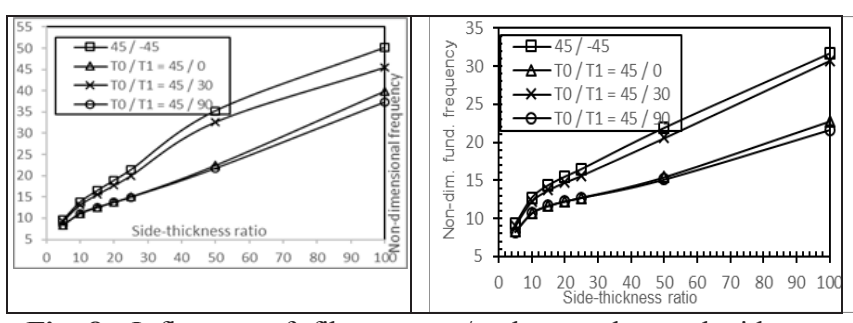

Fig 8: Influence of fibre centre/ edge angles and side-tothickness ratio on the fund. freq. of a 2-layered square supported spherical (left) and cylindrical (right) shell, a/h=10; $\mathrm{R} / \mathrm{a}=20$

The frequency of shells varies, almost linearly, with side-to-thickness ratio even beyond $\mathrm{S} \geq 20$ unlike plates. The frequency variation in a 3-layered spherical shell is similar to 2-layered configuration and hence not presented. It is interesting to note that the influence of fibre angles is predominant for very thin shells rather than thicker ones. The increase in fibre angle at the edge for the minimum centre angle $\left(\mathrm{T} 0=0^{\circ}\right)$, results in increase of the frequency while the angle at the edge has very little influence at the maximum centre angle $\left(\mathrm{T} 0=90^{\circ}\right)$. The frequency appears to increase/decrease with the increase in the angle at the fibre edge for intermediate 
values of centre angle. Similar observations are true for laminates with constant edge angle and increase in the centre angle. The change in frequency of 2/3-layered cylindrical shells is qualitatively similar to the spherical shells.

\subsubsection{Influence of aspect ratio}

The variation of frequencies with aspect ratio for 2layered cylindrical shell is presented in Figure 9. The frequency in cylindrical shells increases with aspect ratio though the rate of increase is steep in shells with high $a / b$ ratio. The impact of fibre angles on the frequency is not significant at minimum/maximum fibre centre angle $\left(\mathrm{T} 0=0^{\circ}\right.$ or $\left.90^{\circ}\right)$. The extremities of edge angle $\left(0^{\circ}\right.$ and $90^{\circ}$ ) results in almost similar frequency variation while the frequency is maximum for the edge angle of $45^{\circ}$.

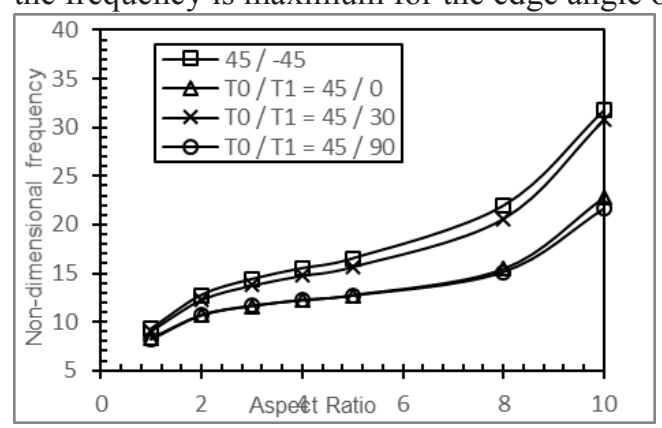

Fig 9: Influence of fibre centre/ edge angles and aspect ratio on the natural frequencies of a 2-layered square simply supported cylindrical shell, $\mathrm{a} / \mathrm{h}=10 ; \mathrm{R} / \mathrm{a}=20$

\subsubsection{Influence of radius-to-thickness ratio}

The effect of fibre angles and the radius-to-thickness ratio on the frequency of the 2-layered spherical shells is presented in Figure. 10. The frequency, in general, decreases with the increase in the $\mathrm{R} / \mathrm{h}$ ratio till it reaches 35 and remains fairly constant thereafter. There is no $t a$ visible change in frequencies at the extremities of centre angle $\left(0^{\circ} / 90^{\circ}\right)$. The frequency increases with the increase in the fibre angle at the edge reaches $45^{\circ}$ and drops with additional increase in the edge angle.

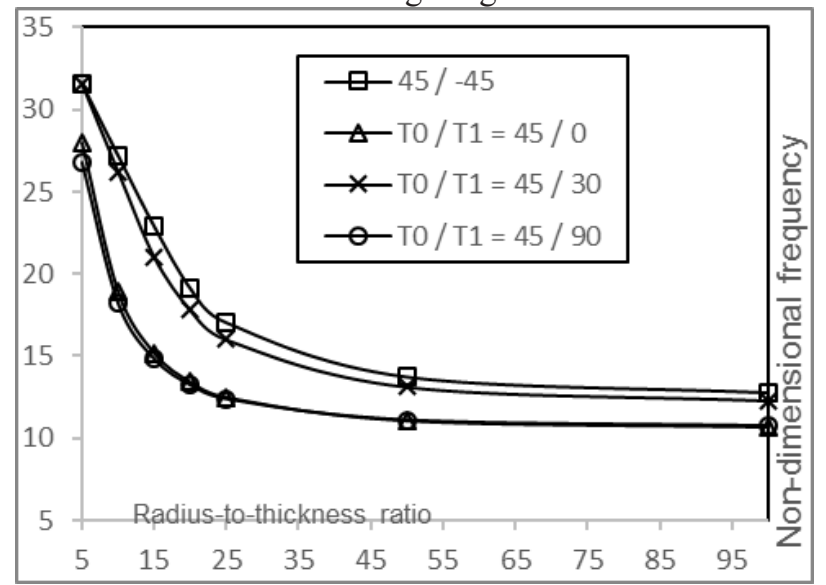

Fig 10: Influence of R/h ratio on the frequencies of a 2-layered square simply supported spherical shell, $\mathrm{a} / \mathrm{h}=10$;

\subsubsection{Impact of edge conditions}

The influence of simply supported/clamped edge conditions on the spherical shell is shown in Figure. 11. The frequency for a clamped shell is higher than the SS laminate, as expected. The frequency of the shell increases with thickness ratio as the decrease in mass is quite drastic than the lessening in the stiffness.

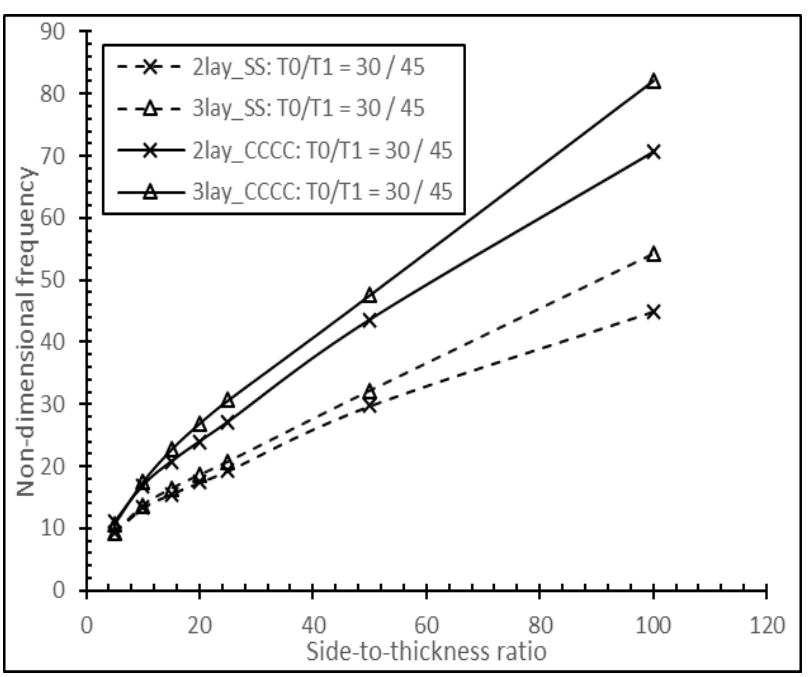

Fig 11: Influence of edge conditions on the natural frequencies of a 2-layered square simply supported square spherical shell

\subsubsection{Impact of moisture concentraion}

The impact of moisture on the frequency against the shell radius-to-thickness ratio is shown in Figure. 12 for shell with $\mathrm{a} / \mathrm{h}=10$ and for three different configurations (2/3/8 layers). The frequency decreases with increase in moisture due to decrease in the overall stiffness values of the shell as expected. The presence of moisture has a greater impact on the eigenvalue of a thick shell than a thin shell.

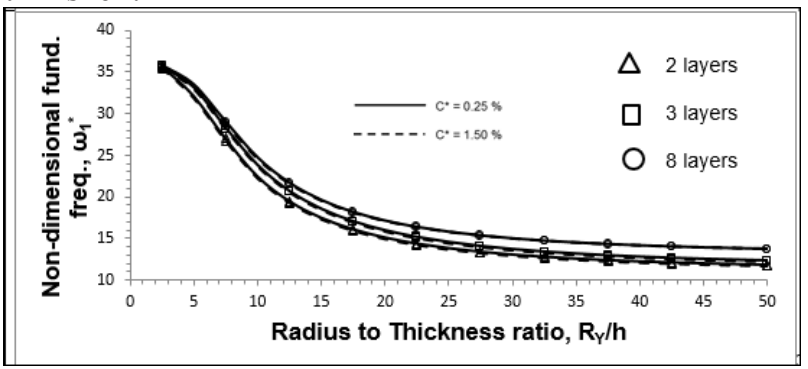

Fig 12: Influence of moisture on the frequencies of a $2 / 3 / 8$ layered square simply supported square spherical shell with $\mathrm{T} 0 / \mathrm{T} 1=30^{\circ} / 45^{\circ}$

\subsubsection{Mode shapes of spherical and cylindrical shells}

The second mode shape of 2-layered spherical and cylindrical shells with $\mathrm{T} 0 / \mathrm{T} 1=0^{\circ} / 45^{\circ}$ and thickness 
ratio $(\mathrm{a} / \mathrm{h})$ of 10 is shown in Figure 13 . It can be noted that fibre angles alter the deformed shapes of higher order modes.

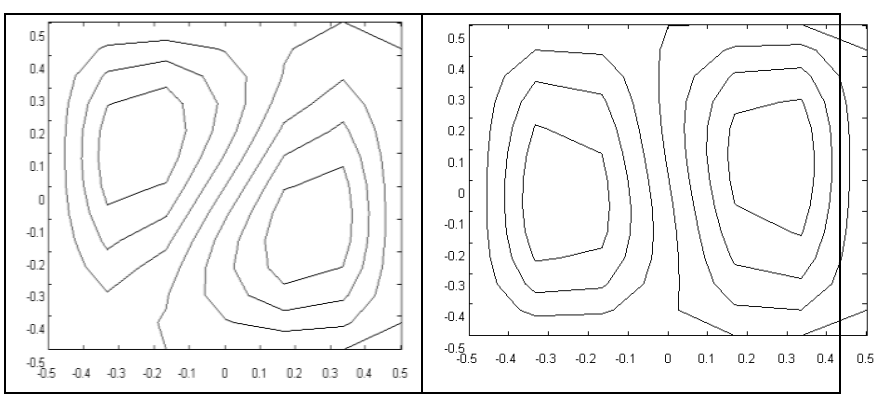

Fig 13: Third mode shapes of 2-layered spherical (left) and cylindrical (right) for a curved fibre $\mathrm{T} 0 / \mathrm{T} 1=0^{\circ} / 45^{\circ} ; \mathrm{a} / \mathrm{h}=10$

\section{Conclusions}

The following conclusion can be drawn from the present study on linear free vibration:

- The frequencies of shells match closely with the published results.

- The frequency of shells decreases with increase in centre angle for negative edge angles for a 2-layered plate while centre angle influences the frequency in the 3-layered configuration for positive and negative fibre edge angles. The maximum/minimum frequencies in 2/3-layered plates occur for different fibre angles.

- The frequency increases with layers and fibre angle have varying degree of influence on the frequency of plates/shells.

- The frequency of spherical/cylindrical shells increases linearly with the increase in side-to-thickness even beyond 20 .

- The frequency of shells increases with the aspect ratio though the rate of increase is different for different aspect ratios.

- The extremities of the angle at the edge $\left(30^{\circ} / 90^{\circ}\right)$ results in almost similar frequency for plates/shells while the frequency is maximum for the edge angle of $45^{\circ}$.

- The frequency of the clamped shells is higher than the simply supported laminates.

- The presence of moisture and the temperature is observed to be significant for thick shells rather than for moderate/thin shells.

Bibliography:

[1] B. C. Kim, P. M. Weaver, and K. Potter, "Manufacturing characteristics of the continuous tow shearing method for manufacturing of variable angle tow composites," Compos. Part A Appl. Sci. Manuf., vol. 61, no. 8, pp. 141-151, (2014).

[2] T. Kant and K. Swaminathan, "Analytical solutions for the static analysis of laminated composite and sandwich plates based on a higher order refined theory," vol. 56, pp. 329-344, (2002).

[3] M. M. Abdalla, S. Setoodeh, and Z. Gürdal, "Design of variable stiffness composite panels
[4]

for maximum fundamental frequency using lamination parameters," Compos. Struct., vol. 81, no. 2, pp. 283-291, (2007). laminated fibrous composite plates with local anisotropy induced by short fibers and curvilinear fibers," Compos. Struct., vol. 93, no. 2, pp. 902-910, (2011).

[5] A. W. Blom, S. Setoodeh, J. M. A. M. Hol, and Z. Gürdal, "Design of variable-stiffness conical shells for maximum fundamental eigenfrequency," Comput. Struct., vol. 86, no. 9, pp. 870-878, (2008).

[6] H.-C. Lo and M. W. Hyer, "Fundamental frequencies of elliptical composite cylinders with circumferentially varying fiber orientation," AIAA J., vol. 50, no. 4, pp. 928-937, (2012).

[7] P. Ribeiro, "Linear modes of vibration of cylindrical shells in composite laminates reinforced by curvilinear fibres," J. Vib. Control, vol. 22, no. 20, pp. 4141-4158, Mar. (2015).

[8] A. Houmat, "Nonlinear free vibration analysis of variable stiffness symmetric skew laminates," Eur. J. Mech. - A/Solids, vol. 50, pp. 70-75, (2015)

[9] M. Ganapathi and D. P. Makhecha, "Free vibration analysis of multi-layered composite laminates based on an accurate higher-order theory," Compos. Part BEngineering, vol. 32, no. 6, pp. 535-543, (2001).

[10] L. Piegl and W. Tiller, "The NURBS Book," Computer-Aided Design, vol. 28, no. 8. pp. 665666, (1996).

[11] N. Grover, B. N. Singh, and D. K. Maiti, "An inverse trigonometric shear deformation theory for supersonic flutter characteristics of multilayered composite plates," Aerosp. Sci. Technol., vol. 52, pp. 41-51, (2016).

[12] J. N. Reddy and C. F. Liu, "A Higher-Order Theory for Geometrically Nonlinear Analysis of Composite Laminates," NASA Contract. Rep. 4056, (1987). 